\title{
Ueber das Excretin;
}

\section{von Dr. Friedrich Hinterberger.}

(Der k. Acad. der Wissenschaften zu Wien vorgelegt in der Sitzung am 10. October 1872.)

Das Excretin wurde von Marcet*) in den Excrementen des Menschen entdeckt und für einen schwefelhaltigen Körper von der Formel $\mathrm{C}_{78} \mathrm{H}_{156} \mathrm{SO}_{2}$ erklärt.

Bei einer Untersuchung meiner frischen Excremente, welche ich drei Jahre fortsetzte, erhielt ich von 100 Pfund der frischen Excremente $8 \mathrm{Grm}$. reines Excretin. Das Excretin der verschiedenen Darstellungsweisen enthielt manchmal 2,8 und auch mehr Procente Scliwefel und häufig 0,6 bis $7 \mathrm{pC}$. Stickstoff. Dieses Verhalten, sowie der Umstand, dafs meine Analysen mit der Analyse M a r c e t's nicht stimmten, brachten mich zu der Vermuthung, dafs man es hier mit einem unreinen Körper zu thun habe.

Es ist mir in der That gelungen, das Excretin frei von Sclıwefel und Slickstoff zu erhalten und mit Hülfe einer Bromverbindung die empirische Formel des Excretins festzustellen. Die Darstellung bestand in Folgendem : $214 \mathrm{Grm}$. der frischen Excremente **) wurden täglich mit $350 \mathrm{CC}$. Weingeist von $90 \mathrm{Vol}-\mathrm{pC}$. ausgekocht. Hierzu diente eine Flasche von Weifsblech von $16 \mathrm{Ctm}$. Höhe und $11 \mathrm{Ctm}$. Durchmesser, welche mit einem Rückflufskühler verbunden war. Die erhaltene weingeistige Lösung wurde auf ein faltiges Filter gegossen und der Rückstand in der Flasche nochmals mit $175 \mathrm{CC}$. Weingeist von 90 Vol.-pC. ausgezogen. Die weingeistige Lösung ist dunkelbraun und setzt nach achttägigem Stehen einen Niederschlag $a b$, welcher im trockenen Zustande beinahe schwarz ist. Dieser Niederschlag besteht aus Excretin und dem Magnesiumsalze einer Gallensäure oder einer neuen Säure. Dieses Magnesiumsalz ist sehr schwer in Weingeist löslich, läfst sich durch Krystallisation von dem leicht löslichen Excretin trennen und lieferte bis jetzt die Formel

$$
\mathrm{C}_{56} \mathrm{H}_{113} \mathrm{MgNO}_{11} \text {. }
$$

Wenn es sich darum handelt, reines Excretin darzustellen, bekümmert man sich nicht um den Niederschlag, welcher sich freiwillig aus der weingeistigen Lösung der Excremente absetzt.

*) Chem. Centralblatt 1860, 586; Gmelin's Handb. 7, 2193.

**) 214 Grm. ist das Mittel von 130 gewogenen Stühlen. 
Man sammelt vielmehr diesen Niederschlag auf einem Filter, versetzt das Filtrat mit $20 \mathrm{CC}$. Kalkmilch, welche etwa 1,5 Grm. Kalk enthält und verdünnt die erhaltene Flüssigkeit mit $500 \mathrm{CC}$. Wasser. Es entsteht ein lichtbrauner Niederschlag, welcher neben anderen Substanzen das Excretin enthält. Dieser Niederschlag wird nach 24 Stunden auf einem Filter gesammelt, mit Wasser ausgewaschen und an der luft getrocknet.

Von 38 Pfund der frischen Excremente erhält man $142 \mathrm{Grm}$. des Kalkniederschlages, welcher das Excretin enthält.

$35 \mathrm{Grm}$. des lufttrockenen Kalkniederschlages kocht man in einer Blechflasche unter Anwendung des Rückflufskühlers mit einer Mischung von $75 \mathrm{CC}$. Weingeist von $90 \mathrm{Vol}-\mathrm{pC}$. und 75 CC. Aether, filtrirt und kocht den ungelösten Rückstand abermals mit $75 \mathrm{CC}$. Weingeist und $75 \mathrm{CC}$. Aether aus.

Läfst man das gelbe Filtrat acht Tage bei einer Temperatur unter $0^{0}$ ruhig stehen, so scheidet sich alles Excretin in Form von gelben nadelförmigen Krystallen ab, welche zu halbkugelartigen Gruppen vercinigl sind.

Dieses rohe Excretin sammelt man anf einem Filter, waseht es nicht mit Weingeist aus und trocknet is, ohne zll pressen, an der Luft.

Hat man etwa 5 Grm. des rohen Excretins, so reinigt man dasselbe durch Uinkrystallisiren. Hierzu benutzt man Weingeist von $95 \mathrm{~V}$ ol.-pC. und Blutkohle, wiederholt dasselbe 4 bis 5 mal und sorgt dafür, dafs die Krystallisation immer bei einer Temperatur unter $0^{\circ}$ vor sich geht.

Erhitzt man das so erhaltene Excretin mit Kalium in einer kleinen Eprouvette und behandelt den Rückstand nach dem Verbrennen des Kaliums mit wenig Wasser, so erhält man eine Lösung, welche eine verdünnte Lösung von Nitroprussidnatrium nicht violett färbt, mithin frei von Schwefel ist.

Unreines schwefelhaltiges Excretin läfst sich auch durch Destillation mit der zehnfachen Menge Kalk schwefelfrei darstellen, scheint aber hierbei die Eigenschaft, zu krystallisiren, einzubüfsen, obne seine Zusammensetzung zu ändern.

Behufs der Elementaranalyse wurde das Excretin über Schwefelsäure getrocknet und im Platinschiffchen unter schlicfslicher Anwendung von Sauterstoffgas verbrannt.

I. 0,3024 Grm. Substanz gaben 0,9035 Kohlensäure und $\mathbf{0 , 3 3 7 4}$ Wasser.

II. 0,3534 Grm. Substan gaben 1,057 Kohlensäure und 0,401 Wasser.

III. 0,3209 Grm. Substanz gaben 0,9692 Kohlensüure und 0,361 Wasser. 
Aus diesen Daten berechnet sich als einfachste Formel $\mathrm{C}_{20} \mathrm{H}_{36} \mathrm{O}$, nach welcher sich Rechmung und Versuch in folgender Weise vergleichen :

\begin{tabular}{lccccc} 
& & \multicolumn{3}{c}{ Gefunden } & Im Mittel \\
\cline { 3 - 5 } $\mathrm{C}_{20}$ & 82,19 & I. & II. & III. & 81,81 \\
$\mathrm{H}_{36}$ & 12,33 & 1,48 & 81,57 & 82,37 & 12,50 \\
$\mathrm{O}$ & 5,48 & - & - & - & -
\end{tabular}

Das Cholestearin $\mathrm{C}_{26} \mathrm{H}_{44} \mathrm{O}$ kommt dem Excretin in der procentischen Zusammensetzung nahe. Das Cholestearin löst sich aber schwerer in Eisessig, als das Excretin. Die Lösung des Cholestearins in Eisessig zeigt unter dem Mikroscope schöne seideglänzende sechsseitige Prismen *), während die Lösung des Excretins in Eisessig kugelige Massen bildet. Das Cholestearin giebt mit Brom ein Substitutionsproduct mit 7 Atom Brom und ein Additionsproduct mit 2 Atom Brom **), währen das Excretin sich in folgender Weise verhält.

Behandelt man das Excretin mit Brom, so entstelıt unter Erwärmung und Entwickelung von Bromwasserstoff tine schwarzbraune Flüssigkeit, welche beim Uebergiefsen mit Aether zu einer braunen harzartigen Masst zusammenballt. Kocht man diese Masse nach dem Verjagen des freien Broms mit einer Mischung von Alkohol und Aether, so löst sie sich. Die Lösung scheidet beim freiwilligen Verdunsten harte spröde, zu Kugeln vereinigte Krystalle ab, welche durch Umkrystallisiren farblos werden. Das erhaltene Bromproduct ist unlöslich in Wasser, schwer löslich in Weingeist, leicht löslich in alkoholhaltigem Acther und schmilzt beim Erhitzen im Wasserbade.

Dic bei $100^{\prime \prime}$ getrocknete Substanz wurde mit cliromsaurem Blei verbrannt und zur Ermittelung des Bromgehaltes mit Kalk geglüht.

I. 0,4746 Grm. Substanz gaben 0,9388 Kohlensäure und 0,3271 Wasser.

II. 0,3574 Grm. Substanz gaben 0,298 Bromsilber.

Diese Analysen fübren zur Formel $\mathrm{C}_{20} \mathrm{H}_{34} \mathrm{Br}_{2} \mathrm{O}$, wie folgende Zusammenstellung zeigt :

*) Diese Annalen 187, 107.

**) Daselbst 146,175 . 


\begin{tabular}{lccc} 
& Berechnet & \multicolumn{2}{c}{ Gefunden } \\
$\mathrm{C}_{\mathbf{2 0}}$ & 53,33 & $\mathbf{5 3 , 9 4}$ & II. \\
$\mathrm{H}_{34}$ & $\mathbf{7 , 5 5}$ & $\mathbf{7 , 6 5}$ & - \\
$\mathrm{Br}_{\mathbf{2}}$ & $\mathbf{3 5 , 5 5}$ & - & 35,47 \\
$\mathrm{O}$ & $\mathbf{3 , 5 7}$ & - & -
\end{tabular}

Während es mithin leicht ist, aus dem Excretin ein krystallisirtes Bibromexcretin darzustellen, gelang es nicht, das Chlorexcretin zu bereiten. Versetzt man nämlich die weingeistige Lösung des Excretins mit Wasser, fügt zur erhaltenen Emulsion Chlorwasser und schüttelt, so erhält man beim Verdunsten eine farblose syrupartige Masse.

Es ging mir leider das Material aus, um weitere Versuche anzustellen, um über die Structur des Excretins ins Reine zu kommen. Ich kann daher unterdessen nur die empirische Formel des reinen Excretins $\mathrm{C}_{20} \mathrm{H}_{36} \mathrm{O}$ und die Formel des daraus dargestellten Bibromexcretins $\mathrm{C}_{20} \mathrm{H}_{34} \mathrm{Br}_{2} \mathrm{O}$ als Endresultate dieser Arbeit anführen. 
Annalen der Chemie u. Pharm. Bd. CLXVI. Taf.II.

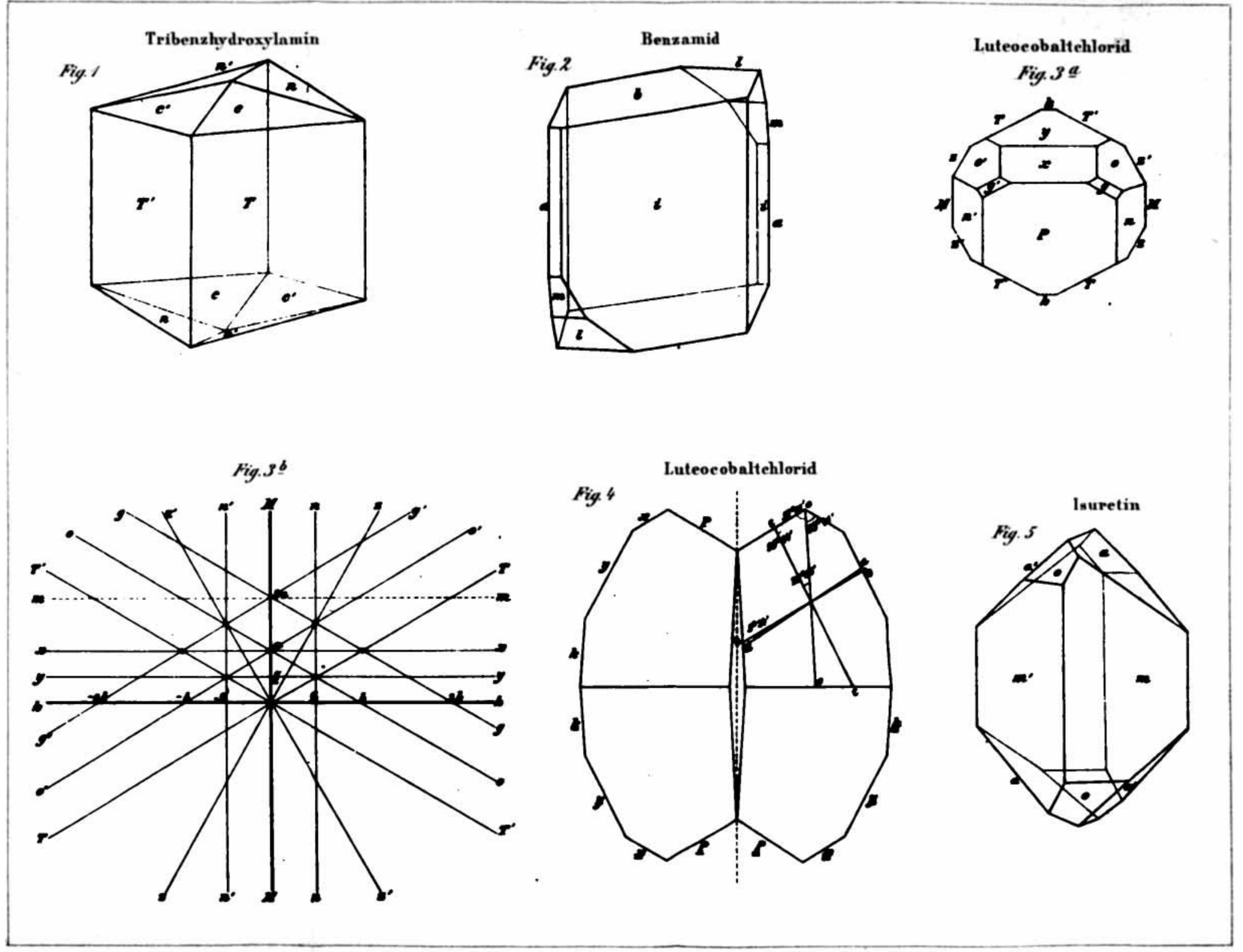

